\title{
A JOINT NUMERICAL AND EXPERIMENTAL STUDY OF THE JET OF AN AIRCRAFT ENGINE INSTALLATION WITH ADVANCED TECHNIQUES
}

\section{Brunet, P. Molton, H. Bézard, S. Deck, and L. Jacquin}

ONERA, Meudon

Rue des Vertugadins 8, Meudon 92190, France

\begin{abstract}
This paper describes the results obtained during the European Union JEDI (JEt Development Investigations) project carried out in cooperation between ONERA and Airbus. The aim of these studies was first to acquire a complete database of a modern-type engine jet installation set under a wall-to-wall swept wing in various transonic flow conditions. Interactions between the engine jet, the pylon, and the wing were studied thanks to "advanced" measurement techniques. In parallel, accurate Reynolds-averaged Navier-Stokes (RANS) simulations were carried out from simple ones with the Spalart-Allmaras model to more complex ones like the DRSM-SSG (Differential Reynolds Stress Modef of SpezialeSarkar-Gatski) turbulence model. In the end, Zonal-Detached Eddy Simulations (Z-DES) were also performed to compare different simulation techniques. All numerical results are accurately validated thanks to the experimental database acquired in parallel. This complete and complex study of modern civil aircraft engine installation allowed many upgrades in understanding and simulation methods to be obtained. Furthermore, a setup for engine jet installation studies has been validated for possible future works in the $\mathrm{S} 3 \mathrm{Ch}$ transonic research wind-tunnel. The main conclusions are summed up in this paper.
\end{abstract}

\section{INTRODUCTION}

The diameter of the turbofan engines for civil transport aircraft has increased in the last decades to improve the propulsion efficiency and reduce the fuel consumption. Even for long range aircraft, for which two big engines or four smaller engines are proposed, the two-engine versions are often selected for global economic reasons. This tendency, coupled with the different constraints at aircraft level (ground clearance, short pylon preferable for heavy engines for structural reasons, etc.), leads to relative engine positions closer to the wing. For the next

This is an Open Access article distributed under the terms of the Creative Commons Attribution-Noncommercial License 3.0, which permits unrestricted use, distribution, and reproduction in any noncommercial medium, provided the original work is properly cited. 
generation of aircraft projects, the intensity of the interactions between the engine and the airframe could be important and will generate critical phenomena. In addition, when the engine is installed closer and closer to the airframe, the evolutions of the interactions are no more linear and it is difficult to extrapolate the actual conventional solutions in term of engine installation.

Airbus has identified specific topics to be investigated to overcome these possible concerns. The analysis of the interactions between the engine jet and the airframe is one of these topics. So, Airbus and ONERA have decided to launch the research project JEDI and have defined the detailed activities of this paper. It was decided to perform in parallel numerical and experimental activities in this project, with tests in the ONERA S3Ch Chalais-Meudon wind-tunnel, and to investigate mainly the aerodynamic phenomena.

The studied configuration is composed of a wall-to-wall swept wing with engine installation, representing a modern one of an Airbus-type aircraft. An air supply system was adapted upstream of the nacelle in order to provide pressurized air for the fan and core jets and to perform wind-tunnel tests with a hot core jet. A boundary layer suction system was integrated in order to diminish the incoming boundary layer from the air supply stick.

As for experimental activities in the transonic S3Ch wind tunnel of ONERA Chalais-Meudon, many different measurement techniques were applied: "simple" pressure taps for pressure acquisition on the wing, Laser Doppler Velocimetry (LDV) in two-component version (2C) and in three-component version (3C), Planar Image Velocimetry (PIV) in $2 \mathrm{C}$ version and temperature measurements in the jet. These measurement techniques allowed an accurate description of the jet development to be characterized. Two different transonic Mach numbers were considered as well as three different pressure ratios for engine jets per Mach number.

As for numerical activities carried out with the ONERA elsA software, very fine structured grids were made first in order to respect Z-DES constraints in the jets. Some adaptation of boundary conditions were performed in order to match very well the experimental conditions, especially concerning the incoming free-stream, its boundary layer, and the pressure ratios in engine jets. Then, simulations with many different RANS turbulence models were carried out as well as Z-DES simulations in order to compare the accuracy of the various simulation techniques and to upgrade the understanding of this complex flow.

\section{EXPERIMENTAL SETUP AND FLOW CONDITIONS}

\subsection{Model Description}

The investigated configuration is a wall-to-wall wing model equipped with a pylon, an engine, and an air supply stick, as presented in Fig. 1. 


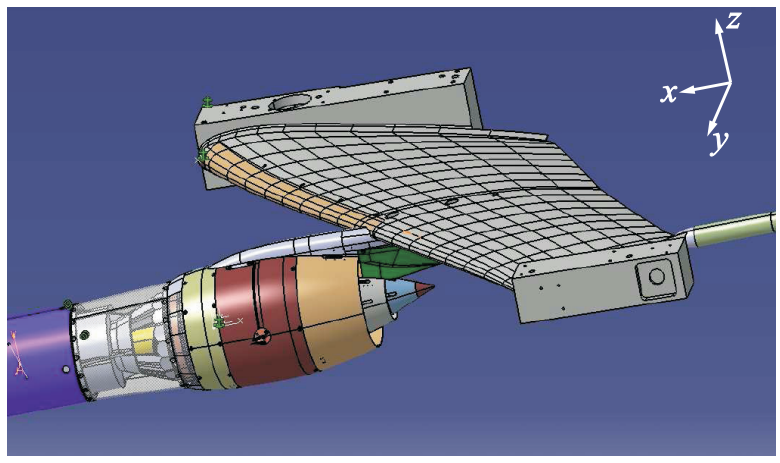

Figure 1 Visualization of the model and the air supply system upstream of the nacelle. (Refer Brunet et al., p. 65.)

The engine installation is slightly different from the one of a present aircraft: the nacelle has a much bigger size relative to the wing and is much closer to the wing. Such configuration represents what could be the engine installation of future aircraft. Specific pylon and nacelle have been manufactured by Airbus France for the JEDI project. The nacelle was studied and manufactured to be able to simulate both the engine fan and the core jets. An air supply system, studied and manufactured at ONERA, was installed upstream of the nacelle and delivered the air for the jets (see Fig. 1). This system allowed variations of the total pressure ratios and of the core jet temperature. A boundary layer develops at the surface of this system and contaminates the nacelle. In order to diminish the thickness of this incoming boundary layer, a suction system has been installed just upstream of the nacelle.

The wing span is $0.8 \mathrm{~m}$, the reference aerodynamic chord is $0.44 \mathrm{~m}$. The diameter of the nacelle $D$ corresponds to $0.1 \mathrm{~m}$ at the trailing edge. Reynolds number based on the aerodynamic mean chord is equal to about 6 million.

\subsection{S3Ch Wind-Tunnel}

The S3Ch wind-tunnel of ONERA Chalais-Meudon is a transonic wind-tunnel operating in atmospheric conditions. The test section is a squared one with a size of roughly $0.8 \times 0.8 \mathrm{~m}$. Specific adapted upper and lower walls have been designed and installed. These fixed three-dimensional (3D) shaped walls have been achieved in order to reduce the wall corrections induced by this transonic configuration. Windows are installed on side walls to allow PIV and LDV measurements to be carried out. 


\subsection{Corrected Flow Conditions and Test Program}

The dimensions of the model are important compared with those of the wind tunnel test section. Six different flow conditions were tested including different nozzle pressure ratios (NPRs) of the jets and they are summed up in Table 1.

Test conditions 1 to 3 were performed at a free-stream Mach number of $0.81 \pm 0.002$ and tests conditions 4 to 6 at a free-stream Mach number of about $0.84 \pm 0.002$.

Table 1 Flow conditions

\begin{tabular}{ccc}
\hline Test condition & NPR $_{\text {FAN }}$ & NPR $_{\text {CORE }}$ \\
\hline 1 & 2.4 & 1.7 \\
2 & 2.2 & 1.6 \\
3 & 2.0 & 1.4 \\
4 & 2.7 & 1.8 \\
5 & 2.4 & 1.7 \\
6 & 2.2 & 1.6 \\
\hline
\end{tabular}

\subsection{Measurements}

All measurement techniques were used for the six flow conditions except LDV 2C and $3 \mathrm{C}$ which were only acquired for the number 4 flow conditions. The widest database was acquired with the LDV $2 \mathrm{C}$ measurement technique and the corresponding measured lines on the engine axis are presented in Fig. 2. Comparisons between the PIV $2 \mathrm{C}$ and LDV $2 \mathrm{C}$ and $3 \mathrm{C}$ measurements are presented in Fig. 3 at the location $x / D=2$ (line 16). One the one hand, $\mathrm{LDV} 2 \mathrm{C}$ and $3 \mathrm{C}$ are in a very good agreement, the grid refinement being higher with LDV $2 \mathrm{C}$ technique.

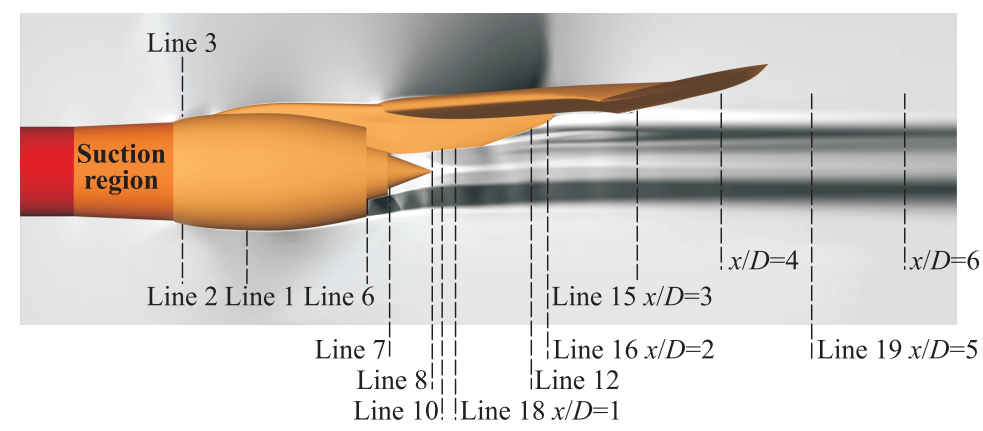

Figure 2 Locations of lines and plans measured around the model. (Refer Brunet et al., p. 66.) 


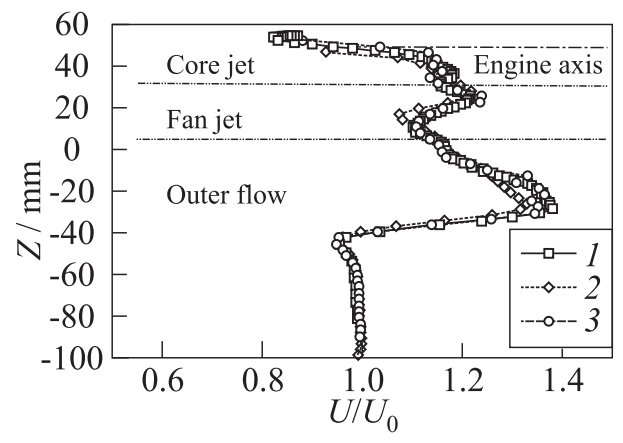

Figure 3 Comparisons between PIV $2 \mathrm{C}$ and LDV $2 \mathrm{C}$ and $3 \mathrm{C}$ at $x / D=2, Y=0 \mathrm{~mm}$ : 1 - LDV 2D; 2 - PIV 2C; and 3 - LDV 3D

On the other hand, some discrepencies can be observed between LDV and PIV, especially in high speed or complex interaction regions.

Measurements presented in this paper are LDV 2C\&3C as well as PIV and, of course, only the flow conditions number 4 .

\section{NUMERICAL SIMULATIONS}

All simulations have been carried out with the structured multiblock elsA software [1]. Numerical methods employed in this study are "classical" ones for aircraft simulations in transonic flow conditions (spatial centred second order scheme of Jameson, implicit LUssor scheme, time accurate second order GEAR scheme with subiterations, backwardeuler + multigrid for steady simulations). Nonetheless, the main turbulence modeling parameters are reminded here since some little modifications were used, especially for Z-DES simulations. This kind of numerical methods for Z-DES simulations was previously validated for transonic buffet studies with the elsA software by Brunet and Deck [2].

\subsection{Reynolds-Averaged Navier-Stokes Turbulence Models}

Several turbulence models were employed in this study. They are reminded below by order of complexity and capability of modeling complex physical phenomena:

(1) the one-equation turbulence model of Spalart-Allmaras [3] called SA in this paper;

(2) the two-equation turbulence model $(k-\omega)$ with shear-stress transport (SST) correction of Menter [4] called SST in this paper; 
(3) the $k$ - $k L$ model [5];

(4) the $k$ - $k L$ Baseline (BSL) model of the DMAE department [6];

(5) the EARSM-WJ (Explicit Algebraic Stress Model of Wallin-Johansson) $k-k L$ model of the DMAE department [5]; and

(6) the DRSM SSG- $\omega$ model $[7,8]$.

Six different flow conditions were simulated with the SA model, others were only used for the flow conditions number 4 .

\subsection{Zonal-Detached Eddy Simulations}

Hybrid RANS/LES (large-eddy simulation) methods during the last years have received increasing attention among turbulence modeling researchers and CFD (computational fluid dynamics) engineers. As an example, the DES method, first introduced in 1997 by Spalart et al. [9] has gained widespread acceptance. The motivation for this approach was to combine the best features of RANS approach and of LES. Reynolds-averaged Navier-Stokes tends to be able to predict attached flows very well with a low computational cost. On the other hand, LES has a high computational cost but allows complex flows prediction to be more accurate.

However, standard DES introduces a significant dependency into the RANS part of the simulation which requires grid spacing for the wall in tangential direction that is larger than the boundary layer thickness at that location. More precisely, if the switch in LES mode occurs inside the RANS boundary layer, this will result in an underestimation of the skin friction coefficient [10]. The grey-area needs careful monitoring but novel approaches are emerging $[11,12]$ to overcome this limitation. To avoid this problem in the incoming attached boundary layer, there was used a Z-DES approach [13, 14] where attached boundary layer regions are explicitly treated in RANS mode regardless to the grid resolution. In effect, the interest of this Z-DES approach is that the user can focus his grid refinement on regions of interest without corrupting the boundary layer properties farther upstream or downstream. Furthermore, it allows to handle as safely as possible complex geometrical configurations.

Concretely, Z-DES has been successfully used to study the mixing enhancement process in a supersonic mixing layer of a sonic underexpanded jet [15] (see [16] for an assessment of the SA model in the frame of axisymmetric underexpanded jets and [17] for a theoretical analysis of the compressible structure of the jet). Then, based on all this experience and on ONERA's "best-practice" for DES type simulations, only Z-DES simulations were carried out in flow conditions number 4 . 


\subsection{Grids}

Two different structured grids were used to perform simulations. The first one is composed of roughly 20 million nodes, mostly concentrated into the jet region. The refinement was adapted in order to match the mixing layers of the jets (as much as possible nodes inside mixing layers), based on first simulations, and no-match joins have been used to reduce the grid size. It was obtained from an iterative process to match the flow solution and to check the grid convergence with RANS modeling. This grid was employed for all RANS simulations as well as for a Z-DES simulation.

Based on this grid and on first Z-DES results, a new refined grid (visible in Fig. 4) was generated in order to solve some refinement problems for the Z-DES simulation. Then this grid is composed of 40 million nodes and it is the reference grid for Z-DES simulation.

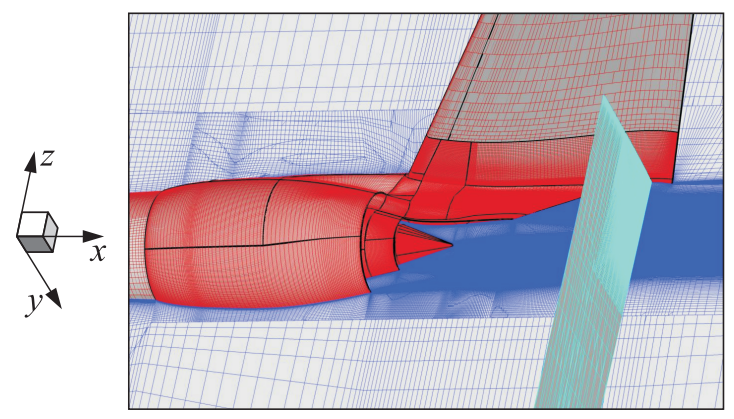

Figure 4 Grid used for Z-DES simulation. (Refer Brunet et al., p. 69.)

\section{ENGINE JET DEVELOPMENT}

Many measurements were acquired during wind-tunnel tests, but only some of them are presented in this paper. Likewise, only number 4 flow conditions corresponding to the higher free stream Mach number and to the higher engine NPR are presented here. These flow conditions were selected for this paper because they correspond to the one with the maximum of experimental and numerical results.

\subsection{Particle Image Velocimetry Measurements}

Three different spanwise vertical plans $(Y=-20 \mathrm{~mm} / 0 \mathrm{~mm} / 20 \mathrm{~mm})$ were acquired with PIV two components (streamwise and vertical components) during 

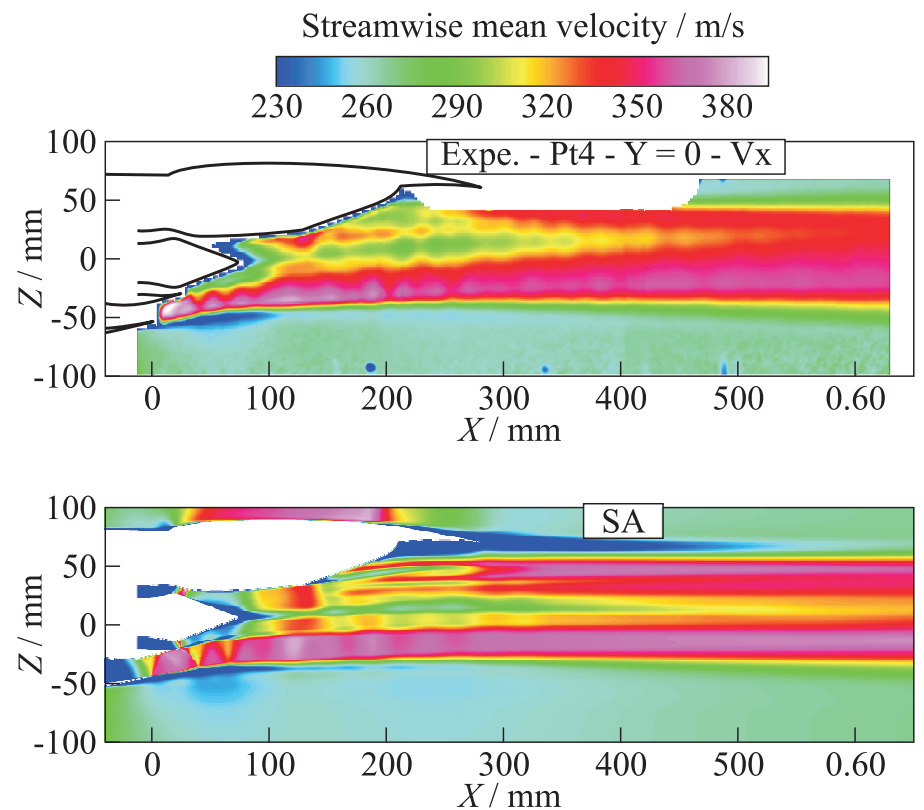

(a)

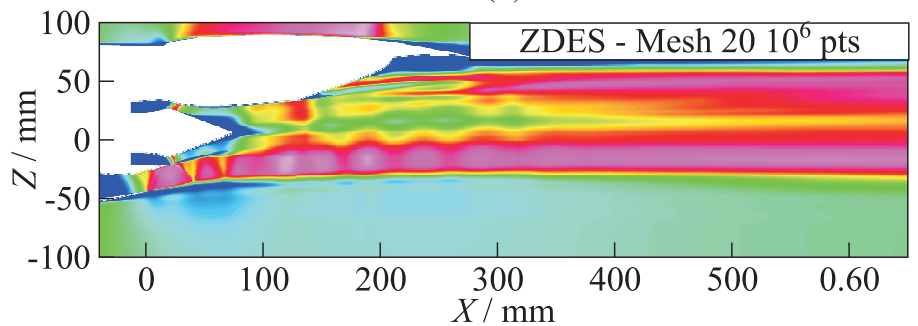

(b)

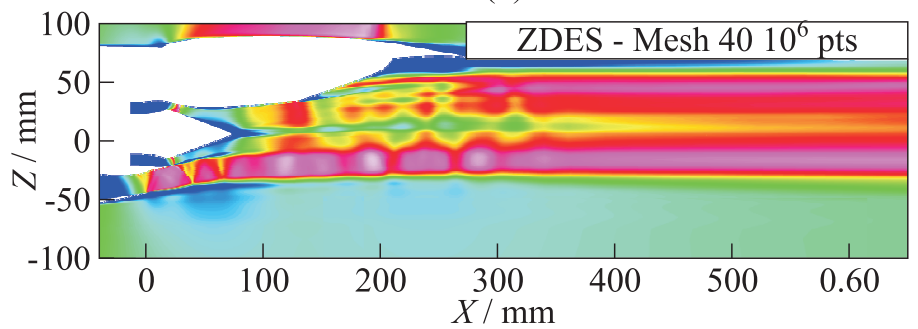

(c)

Figure 5 Streamwise mean velocity fields at mid-span: (a) SA; (b) Z-DES, $20 \cdot 10^{6}$ nodes; and $(c)$ Z-DES, $40 \cdot 10^{6}$ nodes. (Refer Brunet et al., p. 70.) 
the test campaign and for all the flow conditions. As said before, only point 4 flow conditions are presented, and only the streamwise velocity component in the mid-span plan is considered for PIV plans.

Simulations with all the previously described turbulence models present very similar results in all these plans. Then, concerning RANS simulations, the results obtained with the SA turbulence model represent all the RANS models as shown in Fig. 5. In this figure, PIV measurements and Z-DES averaged solutions with the two grids are also plotted. All results are in a quite good agreement and shock cells are more visible with Z-DES simulations, especially with the finest grid. Far from the nacelle, all computed results seem to present a lower diffusion of the jets. These comparisons between experiments and simulations demonstrate that imposed NPRs in simulations are very close to the experimental ones.

The lower part of the jet corresponds to the region the less influenced by the pylon and the wing and it can be roughly considered as an isolated "reference" jet. In this region, the streamwise velocity in the fan jet is clearly higher than the core one which does not present any shock cells. In the upper part of the jet, the interaction between the jets and the pylon shows a complex flow topology downstream of the pylon and it seems to be accurately predicted by all simulations compared with experiments. The beginning of the core jet is not too much influenced by the pylon, but in the wake of the pylon, the fan jet development is complex. Indeed, a low velocity region is observable. This small momentum region is mostly caused by the penetration of the core jet into the pylon wake and then the fan jet is only visible in this axis plane far from the jet exhaust. This analysis can only be seen based on $3 \mathrm{D}$ analysis of the flow (see later in this paper).

\subsection{Laser Doppler Velocimetry Measurements}

To perform more quantitative comparisons including turbulent quantities, LDV two components (streamwise and vertical) measurements were used. These measurements were carried out in vertical lines at mid-span (axis of the engine). First, averaged streamwise velocity is considered. Just downstream of the trailing edge of the nacelle (line 6 - see Fig. 2), in Fig. 6a, all simulations are in a good agreement with measurements. Indeed, for $z<-50 \mathrm{~mm}$, the mean boundary layer profile coming from the nacelle is very well modeled and this showed that the boundary layer suction system is well taken into account into the simulations. Indeed, after some numerical trials, a slipping condition on the entire air supply stick and the suction grid seems to be sufficient to reproduce the averaged measured profiles. Into the fan jet $(z>-50 \mathrm{~mm})$, the exit velocity is accurate even if the profile at the exit is slightly different between experiments and simulations. As for the mixing layer between the fan jet and the outer flow (around $z=-50 \mathrm{~mm}$ ), at that location, it is so thin that only two LDV points 

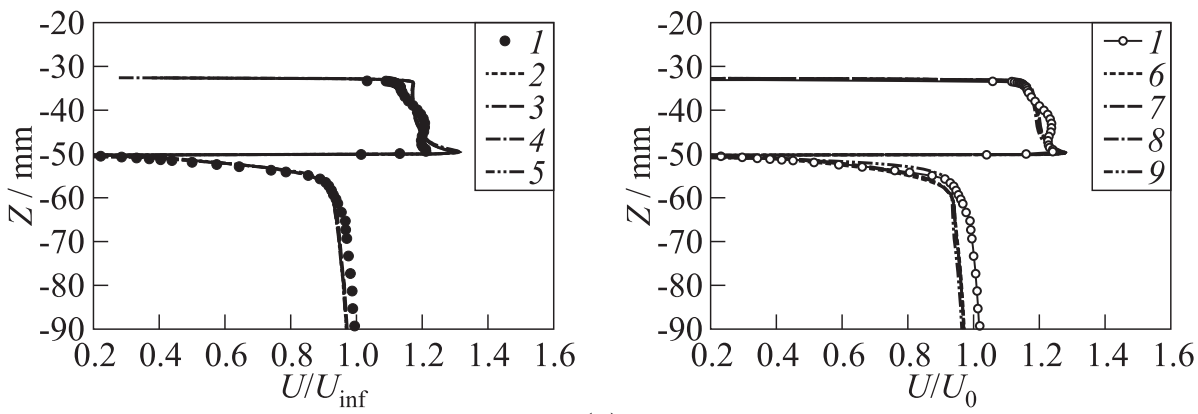

(a)
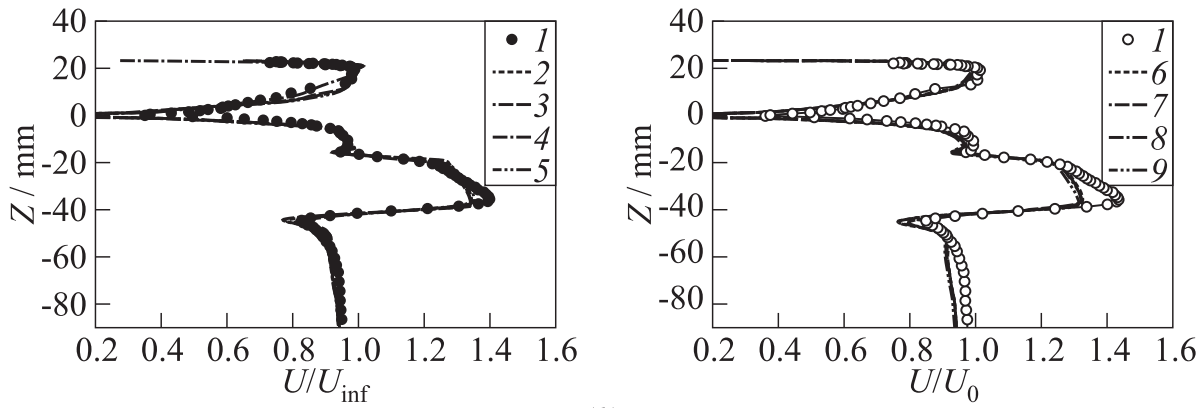

(b)
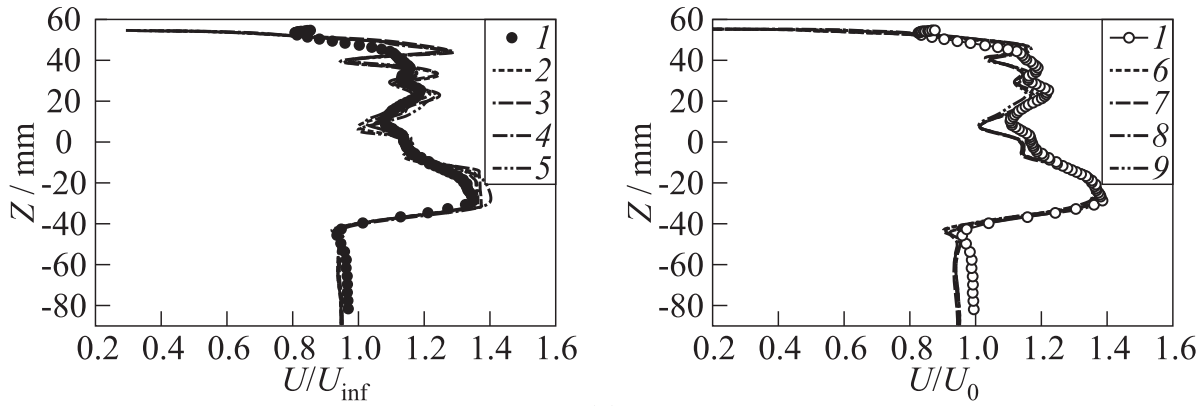

(c)

Figure 6 Streamwise velocity profiles just downstream of the trailing edge of the nacelle (line $6(x=0.2 \mathrm{~mm})$ - see Fig. 2) (a); just downstream of the plug (line 8 $(x=72.5 \mathrm{~mm})$ - see Fig. 2) (b); and far downstream from the nacelle (line 16 $(x=200 \mathrm{~mm})$ - see Fig. 2) (c): 1 - experiments; 2 - SA; 3 - SST; 4 Z-DES, $2 \cdot 10^{6}$ nodes; $5-\mathrm{Z}$-DES, $4 \cdot 10^{6}$ nodes; $6-k$ - $k L ; 7-k-k L$ BSL; $8-$ $k$ - $k L$ EARSM; and $9-\mathrm{DRSM}$ 
are placed inside it and so it is difficult to say if simulations are in a good agreement even if they seem accurate. In these comparisons, no clear difference is observable between all the simulations.

More downstream, at a line located at the trailing edge of the plug (line 8 see Fig. 2), experiments and all simulations are still in a remarkable agreement as presented in Fig. $6 b$. Around the plug trailing edge $(z=0)$, the core jet streamwise velocity seems to be properly predicted. Above of the plug, only the Z-DES simulation with the coarsen grid predicts accurately the profile. This comes from a small separation bubble on the upper side of the plug only computed by this simulation, despite a correct "protection" of attached boundary layer in this region. Then, since this simulation with a separation bubble matches the experiments, one can wonder on the existence of a separation bubble into the experiments. Concerning the fan jet, even if the mean value seems correctly computed, experiments show a more important gradient which could be the consequence of a nonuniform injection into the nacelle. For all simulations, it is interesting to note that the velocity gradients in the mixing layers are very well predicted, showing the quality of the grids and the accuracy of the simulations.

Far from the nacelle (line $16-2 D$ from the trailing edge of the nacelle), differences between experiments and simulations are more evident as presented in Fig. 6c. All simulations present good shapes and levels of the streamwise velocity, but some differences demonstrate the various behaviors of the turbulence models and of Z-DES simulations. Indeed, despite that the mixing layer between the fan jet and the free-stream flow $(z=-40 \mathrm{~mm})$ is very well modeled, the pylon wake and the interaction with the jet show more discrepancies. It is difficult to conclude from the differences between simulations, but it seems evident that the diffusion is less important into simulations since velocity gradients are higher. Considering the relative long distance from the engine exhaust and the too coarsen grid in that region (especially, for Z-DES simulations), one can consider that results are still in a good agreement with experiments.

Concerning turbulent quantities, kinetic turbulent energy measured and computed are presented in Fig. 7 for the three same previous sections. Only simulations with adapted injected turbulence are presented in these plots. Higher levels are linked to the mixing layer between the fan jet and the outer flow and in the pylon wake, but one more time, very close to the fan exit (line 6 $z=-50 \mathrm{~mm}$ ), the mixing layer is very thin and there is not enough LDV points inside to perform very accurate quantitative comparisons. Mixing layer between the core and the fan jets $(z=-33 \mathrm{~mm})$ is rapidly diffused. Except the DRSM model calculation, all simulations are in a remarkable accordance with measurements even if levels are slightly underestimated in the pylon wake. The DRSM model results underestimate turbulent kinetic energy levels and one can notice that the boundary layer coming from the nacelle is laminar.

The shear stress component $u^{\prime} w^{\prime}$ is presented in Fig. 8 for the three selected lines and for SA, SST, and Z-DES simulations. In Fig. $8 a$, the unsteadiness 


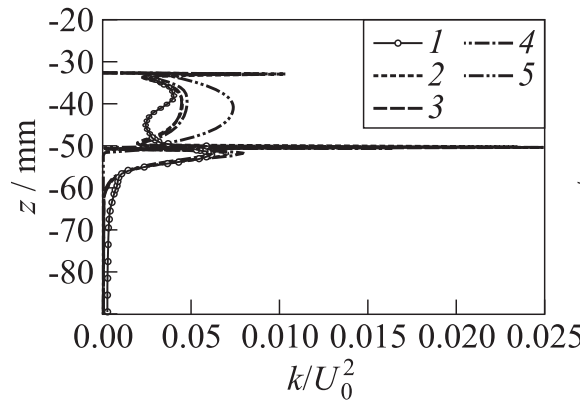

(a)

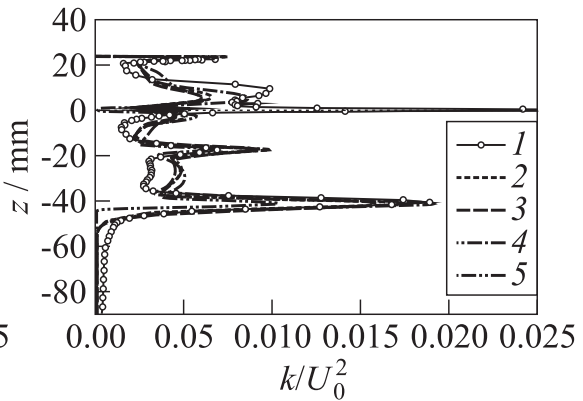

(b)

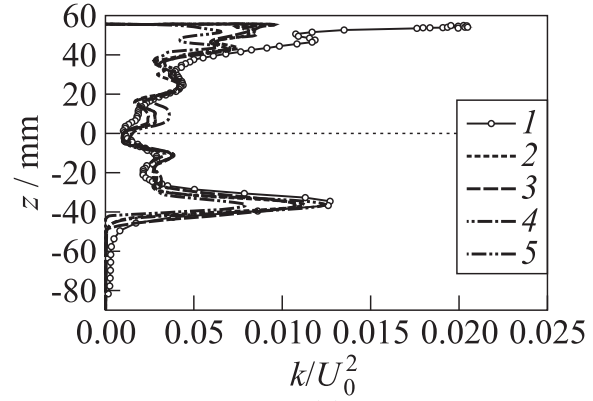

(c)

Figure 7 Turbulent kinetic energy at line $6(x=0.2 \mathrm{~mm})(a)$; line $8(x$ $=72.7 \mathrm{~mm})(b)$; and line $16(x=200 \mathrm{~mm})(c)$ - see Fig. 2: 1 - experiments; $2-k-k L ; 3-k-k L$ BSL; $4-k-k L$ EARSM; and $5-$ DRSM

coming from the boundary layer of the nacelle $(z<-50 \mathrm{~mm})$ is underestimated by all simulations, which could be the consequence of the unsteadiness of the complex boundary layer suction system not taken into account in simulations, despite that the mean profile is correct. Into the fan jet $(y>-50 \mathrm{~mm})$, no turbulence is modeled but the experimental values are quite important. Just downstream of the plug (Fig. 8b), this turbulent quantity is underestimated in the mixing layer between the fan jet and the outer flow by all simulations, especially the Z-DES ones. The mixing layer between the core and fan jets is very well predicted by the Z-DES simulation with the finest grid, overestimated by the other Z-DES simulation and underestimated by RANS simulations. In the trailing edge of the plug, this Reynolds component is always underestimated and above the plug, only the Z-DES with the coarsen grid predicts correct levels, linked to a simulated separation. Far from the nacelle (Fig. 8c), only the mixing layer between the fan and the outer flow and the wake of the pylon are visible and are underestimated by all simulations, but at that location, the grid density is very poor, especially for Z-DES simulations but it should be sufficient for the 


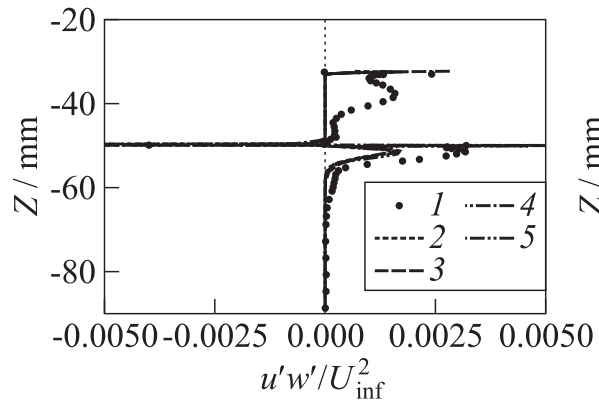

(a)

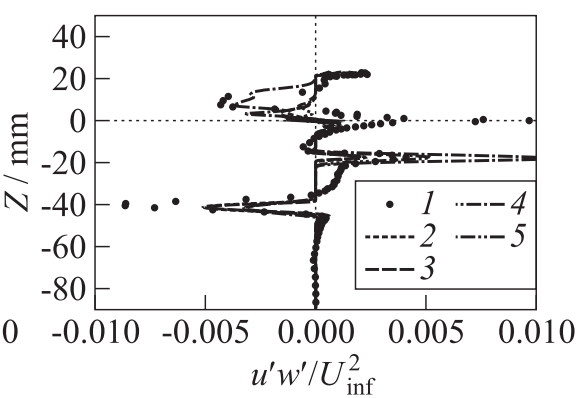

(b)

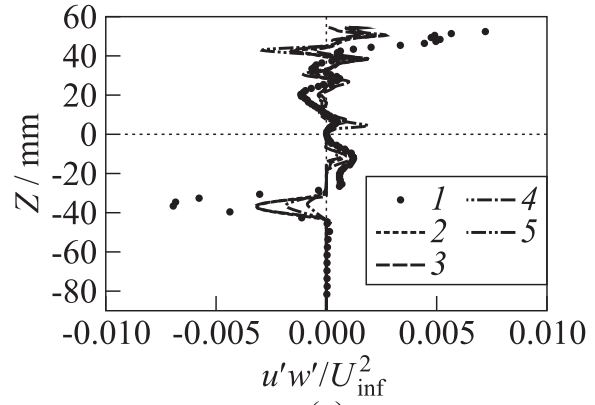

(c)

Figure 8 The $u^{\prime} w^{\prime}$ component at line $6(x=0.2 \mathrm{~mm})(a)$; line $8(x=72.7 \mathrm{~mm})(b)$; and line $16(x=200 \mathrm{~mm})(c)$ - see Fig. 2: 1 - experiments; 2 - SA; 3 - SST; 4 Z-DES $\left(2 \cdot 10^{6}\right.$ nodes $)$; and 5 - Z-DES $\left(4 \cdot 10^{6}\right.$ nodes $)$

RANS ones. For all these curves, SA and SST turbulence models predict more or less the same results.

\subsection{Flow Visualizations with Z-DES Simulations}

It has been shown previously that Z-DES does not allow better results than RANS ones to be obtained if only averaged fields are considered. This also demonstrates that Z-DES simulations allow physical and accurate simulations to be obtained which is not easy on such complex configurations. Moreover, such simulations give a very large amount of information and a direct access to the unsteadiness of the flow. Then, signal processing and more advanced processing could be obtained with such simulations. As an example, in Fig. 9, flow visualization $(\|\operatorname{grad} \rho\|)$ is presented for the Z-DES simulation with the finest grid Schlieren type. This visualization allows turbulent structures, shocks, and acoustics waves to be easily observed. Indeed, in the lower part of the nacelle, 


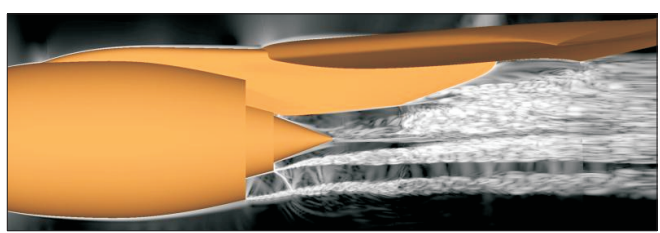

Figure 9 Schlieren-type flow visualizations with Z-DES. (Refer Brunet et al., p. 76.)

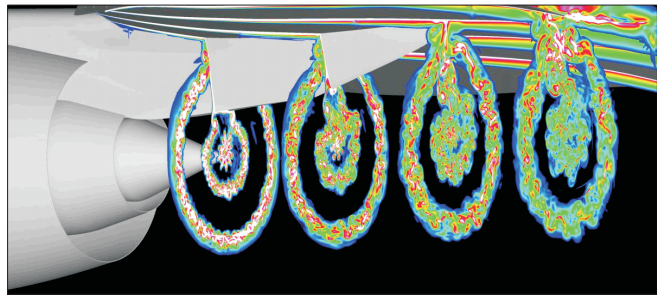

Figure 10 Instantaneous vorticty into the jet obtained by Z-DES simulation (finest grid). (Refer Brunet et al., p. 76.)

turbulent structures into mixing layers are easily visible as well as shock wave in the fan jet. In this region, too, acoustic waves can be observed in the outer flow close to the fan jet. The strong turbulence linked to the interaction between the pylon wake on the jet is also evidenced.

Another flow visualization obtained with the Z-DES simulation on the finest grid is presented in Fig. 10. Four slices into the engine jet are presented with the instantaneous vorticity field. One can remark the 3D behavior of the flow into the mixing layers between the outer flow and the fan jet and between the fan jet and the core jet. The size of the turbulent structures increases with the distance to the engine exhaust. The pylon wake and its complex interaction with the two jets is also clearly visible. As said earlier, the penetration of the core jet into the pylon wake can be evidenced by the vortices that are created. Videos performed based on Figs. 9 and 10 allow the complex 3D turbulent behaviors of the mixing layers and their interactions to be better understood.

\section{CONCLUDING REMARKS AND PERSPECTIVES}

Advanced measurements and simulations have been performed on a realistic engine installation under a swept wing. They allow the complex interactions between the engine jet, the pylon, and the wing to be better understood. Indeed, based on a complex setup in the transonic S3Ch wind-tunnel of ONERA ChalaisMeudon, a wall-to-wall swept wing and engine installation representing modern 
design, many measurement techniques (PSP-TSP-LDV-PIV) were employed in different flow conditions. Engine jet effects were made thanks to an air supply system including temperature effect of the core jet. The very wide database allows, first, to understand and characterize into detail this kind of flow, and, second, to validate CFD codes. This experimental setup of a realistic engine installation into a research transonic wind tunnel has been validated and could be reused in future engine installation studies.

In parallel, CFD simulations were carried out based on very refined grids. Several RANS turbulence models from simple ones (SA) to more advanced ones (DRSM) were evaluated on this flow. Zonal-DES simulations were also carried out. For all simulations, a remarkable accordance with experiments has been demonstrated.

This complete study will allow in the future very accurate CFD simulations and different nacelle shapes (like chevrons) to be studied accurately.

\section{REFERENCES}

1. Cambier, L., and J. P. Veuillot. 2008. Status of the elsA software for flow simulation and multidisciplinary applications. AIAA Paper No. 2008-664.

2. Brunet, V., and S. Deck. 2008. Zonal-detached eddy simulation of transonic buffet on a civil aircraft type configuration. AIAA Paper No. 2008-4152.

3. Spalart, P., and S. R. Allmaras. 1994. A one-equation turbulent model for aerodynamic flows. La Recherche Aerospatiale 5-21.

4. Menter, F. R. 1994. Two-equation eddy viscosity turbulence model for engineering applications. AIAA J. 32(8):1598-605.

5. Bézard, H., and T. Daris. GOD???. Calibrating the length-scale equation with an explicit algebraic reynolds stress model. ERCOFTAC Symposium (International) on Engineering Turbulence Modelling and Experiments - ETMM6. Eds. W. Rodi and M. Mulas. Elsevier.

6. Bézard, H., and F.X. Vandernoot. 2006. CACV - Tâche 2.1.1.1.1. Conception des entrées d'air, distorsion statique, amélioration du modèle EARSM. ONERA RT 6/09508 DAAP/DMAE - CACV/2/RT/011. Novembre.

7. Speziale, C. G., S. Sarkar, and T. B. Gatski. 1991. Modelling the pressure-strain correlation of turbulence: An invariant dynamical systems approach. J. Fluid Mech. $227: 245-72$.

8. Haase, W., B. Aupoix, U. Bunge, and D. Schwamborn. 2006. FLOWMANIA - a European initiative on flow physics modelling. Notes on Numerical Fluid Mechanics and Multidisciplinary Design. Springer. Vol. 14.

9. Spalart, P., W.H. Jou, M. Strelets, and S.R. Allmaras. 1997. Comments on the feasibility of LES for wings and on a hybrid RANS/LES approach. 1st AFSOR Conference (International) on DNS/LES Proceedings. Ruston. 137-47.

10. Nikitin, N. V., F. Nicoud, B. Wasistho, K. D. Squires, and P. R. Spalart. 2000. An approach to wall modeling in large eddy simulation. Phys. Fluids 12:1669-31. 
11. Menter, F. R., M. Kuntz, and R. Bender. 2003. A scale-adaptive simulation model for turbulent flow predictions. AIAA Paper No. 03-0767.

12. Spalart, P. R., S. Deck, M. L. Shur, K. D. Squires, M. Strelets, and A. Travin. 2006. A new version of detached eddy simulation, resistant to ambiguous grid densities. Theor. Comput. Fluid Dyn. 20:181-95.

13. Deck, S. 2003. Numerical simulation of transonic buffet over a supercritical airfoil. AIAA J. 43(7):1556-66.

14. Deck, S. 2005. Zonal-detached eddy simulation of the flow over a high-lift configuration. AIAA J. 43(11):2372-84.

15. Chauvet, N., S. Deck, and L. Jacquin. 2007. Zonal detached eddy simulation of a controlled propulsive jet. AIAA J. 45(10):2458-73.

16. Chauvet, N., S. Deck, and L. Jacquin. 2007. Numerical study of the mixing enhancement in a supersonic jet. AIAA J. 45(7):1675-87.

17. Chauvet, N., S. Deck, and L. Jacquin. 2007. Shock patterns in a slightly underexpanded sonic jet control by radial injection. Phys. Fluid 19:048104. 